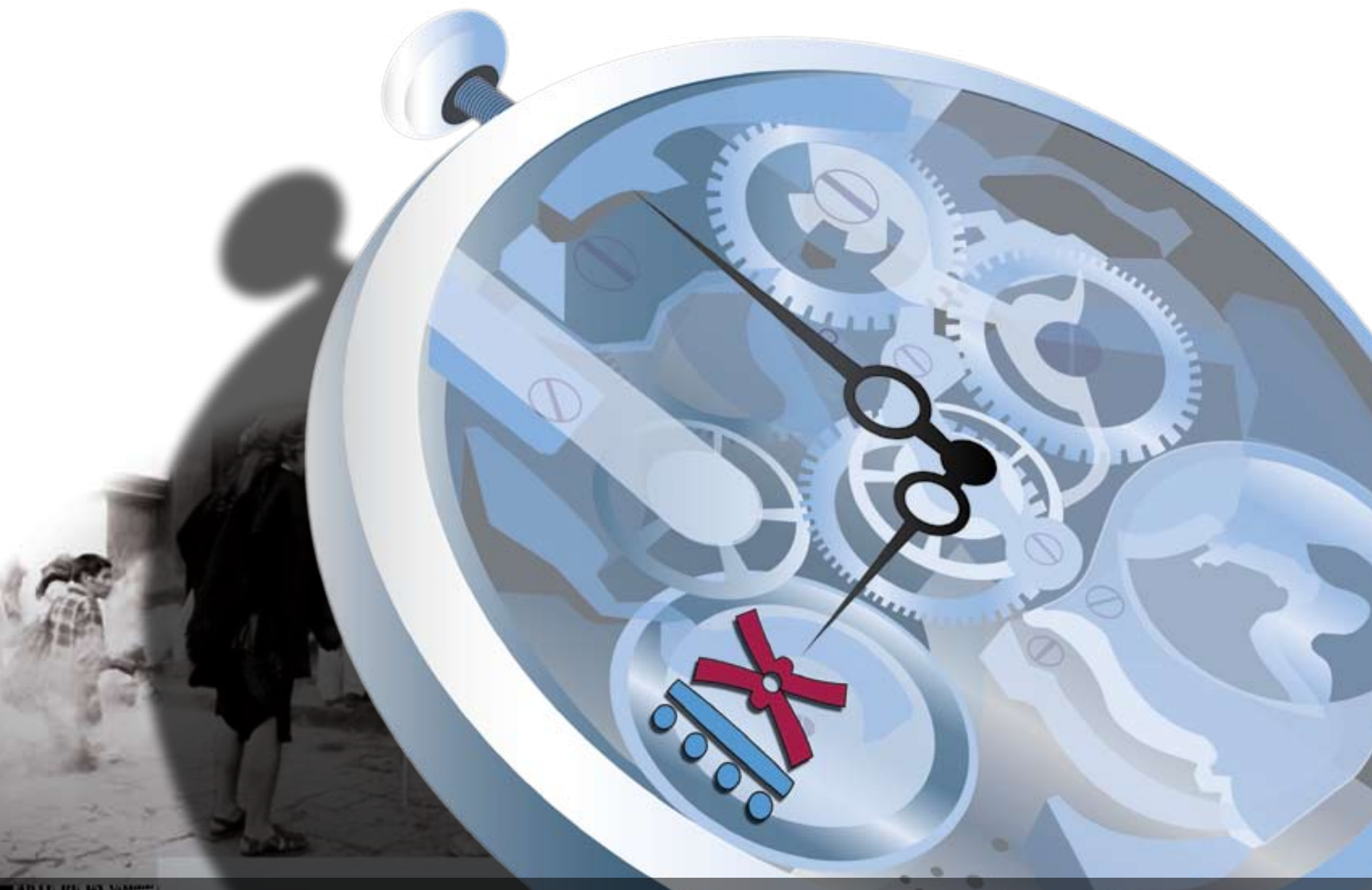

6 $99^{\circ}$ C O N G R S O 2. CENTROAMERICANO DE HISTORIA

Universidad de Costa Rica

ISSN 1409-469X

Fecha de recepción: 15 de mayo 2008 Fecha de aceptación: 30 de mayo 2008

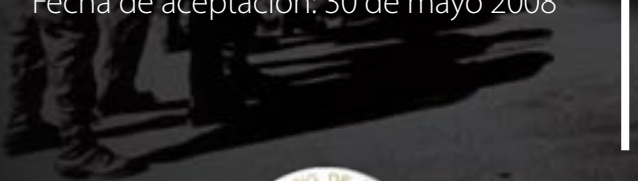

La labor del Comité Cristiano con los refugiados guatemaltecos en Chiapas

Miembros del Consejo Editorial:

Dr. Ronny Viales, Dr. Juan José Marín

Editores Técnicos:

Allan Fonseca, Andrés Cruz, Gabriela Soto
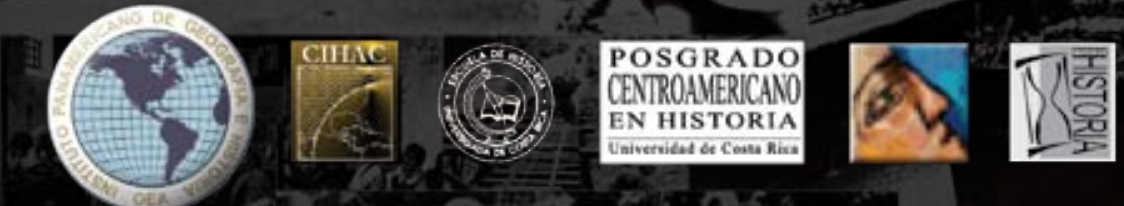
Indexaciones: Repositorio de Revistas UCR, DIALNET, Latindex, REDALYC Directorio y recolector de recursos digitales del Ministerio de Cultura de España, Directory of Open Access Journals. Diálogos Revista Electrónica de Historia ISSN 1409-469X. Número especial 2008. Dirección web: http://historia.fcs.ucr.ac.cr/dialogos.htm

\section{La labor del Comité Cristiano con los refugiados guatemaltecos en Chiapas}

Mtra. Martha Tapia Ramírez

Maestra en Estudios Latinoamericanos por la Facultad de Filosofía y Letras de la UNAM. 
Hacia las décadas de los setentas y ochentas del siglo XX, la política represiva ejercida por los gobiernos de Guatemala generó un éxodo masivo de campesinos hacia la frontera sur de México, estableciéndose la mayoría de ellos en Chiapas.

En ese contexto se reveló que el gobierno de México, a pesar de sostener en el discurso oficial su tradición política de puertas abiertas a perseguidos políticos, manifestó un comportamiento ambiguo. Por un lado, instituyó un organismo para atenderlos: la comisión Mexicana de ayuda a Refugiaos (COMAR), pero, por otro, no fue constante en la asistencia a los refugiados y en ocasiones mostró una actitud de intimidación.

Así, el gobierno mexicano, al no cumplir los requerimientos de los guatemaltecos, dio pie a la fundación de diversas instancias no gubernamentales locales, nacionales e internacionales que brindaron su ayuda. Entre ellas destacó la diócesis de San Cristóbal de las Casas, con la formación del Comité Cristiano, creado en 1979 y dirigido por el obispo Samuel Ruiz García para brindarles asistencia, tema en el que se centra el presente texto. Aquí se mencionan las principales actividades del Comité tanto de asistencia material y espiritual, como de fomento en la organización comunitaria para atender cuestiones de salud, educación y desarrollo productivo. Asimismo, se expone lo relativo al apoyo dado a las organizaciones para el retorno colectivo: las Comisiones Permanentes de Refugiados Guatemaltecos; y la organización de mujeres refugiadas Mamá Maquín.

\section{El Comité Cristiano y su organización}

Desde los albores de los años setenta, la diócesis de San Cristóbal de las Casas se caracterizó por su empeño en ayudar a los pobres dentro de la entidad, como a los de otros lugares. Así recibió a centroamericanos que llegaron huyendo de la situación de guerra de sus países. En 1979, cuando aumentó el número de guatemaltecos que buscaron refugio en Chiapas, la diócesis formó el Comité Cristiano de Solidaridad, conducido por el obispo Samuel Ruiz, con el objeto de brindar apoyo espiritual y material a los recién llegados, para ello contó con el respaldo del Hospital de Comitán y la parroquia de Comalapa. ${ }^{1}$

1 Refugiados guatemaltecos en la diócesis, en Archivo diocesano de San Cristóbal de las Casas, Chiapas, 1989, p. 71, (mimeo); Graciela Freyermuth Enciso y Nancy Godfrey, Refugiados guatemaltecos en México. La vida en un continuo estado de emergencia, México, Centro de Investigaciones y Estudios Superiores en Antropología Social del Sureste (CIESAS)/Instituto Chiapaneco de Cultura, Casa Chata, 1993, p. 32. 
Dentro de la ayuda material del Comité Cristiano en primera instancia, se dieron alimentos básicos, asistencia médica, vivienda y ropa, también se comenzó a hacer saneamiento ambiental. Para ofrecer apoyo a mediano plazo se abrieron proyectos de desarrollo productivo, de defensa legal y política, así como de educación. Un elemento clave brindado por el Comité a los guatemaltecos fue fomentar la organización colectiva de la que algunos ya formaban parte desde su país natal, donde la base estaba en la preservación de su lengua y sus costumbres. Otro de los aportes importantes del Comité Cristiano fue hacer públicas las denuncias de los refugiados con respecto a la violación a los derechos humanos de los que fueron objeto, desde los primeros momentos de su estancia en México y durante el proceso de reubicación; también fue importante el apoyo para el retorno, este último se dio a través de la organización de los propios interesados que formaron las Comisiones Permanentes de Refugiados Guatemaltecos, en donde participaron diversas agrupaciones ya existentes siendo uno de los más destacados el movimiento de mujeres refugiadas Mamá Maquín. ${ }^{2}$

Es importante mencionar que, según fuentes consultadas, ${ }^{3}$ el gobierno mexicano, durante varios años, no permitió oficialmente la ayuda de ONGs nacionales, en buena medida debido a que trataba de evitar la asistencia a los refugiados para que éstos se vieran precisados a regresar pronto a su país. A pesar de ello el Comité Cristiano los ayudó.

El Comité Cristiano tuvo dos etapas, ambas presididas por el obispo Samuel Ruiz. Durante la primera, desde su creación en 1979 hasta 1992, se llamó Comité Cristiano de Solidaridad, tuvo su sede en el seminario de San Cristóbal de las Casas, donde los principales organizadores eran Carlos Casas y Alfredo Inda. Debido a la falta de espacio para atender adecuadamente a los refugiados, aproximadamente en 1987, la sede se cambió a Comitán, donde se compraron tres casas, las cuales se utilizaron respectivamente para oficina administrativa, bodega y aulas para impartir cursos. En la segunda etapa, de 1992 a 1997, el Comité se constituyó como asociación civil llamándose Comité Cristiano para Refugiados A.C., estuvo formado por el vicario Ramón Castillo, el canciller David Méndez Moreno, el vicario de pastoral Gonzalo Ituarte, el vicario

2 Isabel María Martínez Portilla, "La diócesis de San Cristóbal de las Casas y los refugiados guatemaltecos” en Pueblos y Fronteras, núm. 2, Chiapas, noviembre de 2001, pp. 75-79.

3 Entrevista a Hna. Lucía Jiménez Escalona (colaboradora del ex-Comité Cristiano, actualmente atiende a los refugiados guatemaltecos asentados en Chiapas), realizada por Martha Tapia Ramírez en San Cristóbal de Las Casas (SCLC), Chiapas, 23 y 24 de octubre de 2003; Mercedes Oliveira, et. al., Nuestra experiencia ante los retos del futuro. Sistematización del trabajo de las mujeres de Mamá Maquín durante el refugio en México y su retorno a Guatemala, Comitán, ACNUR, 1999, p. 11. 
episcopal Javier Reyes, el cura de Comitán Rodolfo Román y Juan Luis Negrete Fernández como administrador. Las hermanas Lucía Jiménez y Josefina de la Torre y el párroco Javier Ruiz fueron asiduos colaboradores a lo largo de todo el trabajo desempeñado por el Comité. ${ }^{4}$

En el Comité Cristiano en promedio había entre 10 y 20 personas encargadas de base, mientras que eventuales eran aproximadamente 70 individuos mexicanos y extranjeros, entre los que se contaban trabajadores, estudiantes, profesionistas, así como diversos elementos de la iglesia católica que ayudaban temporalmente: sacerdotes, religiosas y miembros de las Comunidades Eclesiales de Base (CEBs), tanto de Chiapas como de otros estados de la república tales como: Nayarit, Jalisco, Coahuila, Guanajuato, Michoacán, Tabasco, D.F., entre otros. También tuvo el constante apoyo de las diócesis de la región Pacífico-Sur y de sedes eclesiásticas fronterizas guatemaltecas como la de El Quiché y Huehuetenango. Los campesinos mexicanos en Chiapas brindaron ayuda fundamental, ya que compartieron casa, comida y tierra, además de brindarles consuelo y seguridad a los refugiados.

La mayor parte del financiamiento económico del Comité Cristiano estuvo a cargo de ONGs internacionales como las siguientes: Adveniat (agrupación a favor de la iglesia en América Latina fundada el 30 de agosto de 1961), Misereor (Organización para el Desarrollo de la Conferencia Episcopal de la Iglesia Católica) y Pan para el mundo, las tres originarias de Alemania; Cebemo (Organización Católica de Cooperación), de Holanda; Manos unidas (Organización para el Desarrollo de filiación católica fundada en 1960), de voluntarios de Italia; Cáritas, de Suiza y Alemania; y Paz y tercer mundo Hirugarren (de Cooperación al Desarrollo, constituida en 1988), en Barcelona. A la disolución del Comité, estas organizaciones continuaron y continúan hasta hoy en día su labor de ayuda en Chiapas. ${ }^{5}$

En los primeros momentos, para lograr una mejor atención a los recién llegados, el Comité Cristiano les pidió la elección de representantes por cada grupo de refugiados para que fungieran como intermediarios entre su comunidad y el Comité, con el fin de facilitar la comunicación y manifestar de forma particular las necesidades de cada conjunto y canalizar la ayuda necesaria. ${ }^{6}$

4 Entrevista a Juan Luis Negrete Fernández, (administrador del ex-Comité Cristiano) realizada por Martha Tapia Ramírez en SCLC, 24 de octubre de 2003.

5 Entrevista a Gerardo González Figueroa (investigador de El Colegio de la Frontera Sur (ECOSUR), actualmente estudia ONGs en Chiapas) realizada por Martha Tapia Ramírez en SCLC, 21 de octubre de 2003; Entrevista a Juan Luis Negrete Fernández, op. cit.

6 “Situación de los refugiados. Trabajo del comité” en El Caminante, núm. 30, Diócesis de San Cristóbal de Las Casas (DSCLC), mayo de 1983, pp. 2-6; “La vida de los campamentos” en El Caminante, núm. 42, DSCLC, abril de 1986, pp. 1-2. 
Los representantes no podían salir a buscar trabajo, pues tenían que quedarse en los campamentos a organizar y repartir la ayuda, así como a recibir y atender a los visitantes y exponerles su situación. También se dedicaban a promover el diálogo tanto entre su comunidad como con las autoridades mexicanas. Su vida estaba en constante peligro porque eran continuamente acosados por el ejército guatemalteco con el fin de evitar la comercialización de su servicio ante la comunidad, trabajaban sin un sueldo regular, su recompensa era la satisfacción de servir a los suyos.

Los representantes iniciales realizaron los primeros estudios "sociológicos" de los campamentos y presentaron al Comité Cristiano estadísticas poblacionales por sectores, edades, etc. A partir de sus informes se pudo saber con cierta precisión cuántos eran, así como cuántos niños ancianos, huérfanos y viudas venían en cada grupo, qué enfermedades padecían, con qué recursos contaban, qué tipo de asistencia inmediata requerían, etc. Sus informes fueron esenciales para planear, solicitar y brindar la ayuda necesaria a las comunidades refugiadas.

\section{Programas de salud y educación}

El Comité Cristiano, en convenio con el Hospital de Comitán, brindó atención médica a los refugiados, desde su llegada. Para el efecto, se organizaron comités de salud que realizaban acciones preventivas y curativas, además de que promovían cursos de medicina tradicional, sobre todo la herbolaria, y también de métodos naturales de control de natalidad. En su seno se formaron promotores de salud, asesorados por la COMAR y otros organismos nacionales.

Para 1989, la diócesis ya contaba con tres hospitales, dos de ellos en zonas marginadas y de difícil acceso: selva y montaña; 15 dispensarios con un doctor o una enfermera cada uno; 413 casas de salud en comunidades rurales a cargo de un promotor de salud, que en total eran unos 719. De acuerdo a estadísticas de la diócesis de San Cristóbal, las personas beneficiadas eran $194565 .^{7}$

A lo largo de las distintas etapas del refugio, esto a través de sus promotores que, como los de salud, ocasionalmente fueron asesorados por la COMAR y otras instancias mexicanas, la educación estuvo a cargo del Comité Cristiano. Ante la inexistente infraestructura, el Comité construyó y habilitó aulas donde se impartín las clases, facilitó libros y material escolar, así como comedores infantiles. Durante varios años fue responsable del seguimiento de los resultados de

7 Refugiados guatemaltecos en la diócesis, op. cit., pp. 79-80. 
los escolares. La mayor parte de los campamentos tenían su propia escuela, llevada por ellos mismos, con excepción de algunos asentamientos con menos de 100 o 150 habitantes.

Los promotores de la educación en su mayoría eran indígenas guatemaltecos, bilingües, pertenecientes a grupos étnicos mayoritarios, su edad promedio era de 25 años, gran parte de ellos eran hombres, generalmente padres de familia, con un bajo nivel de preparación debido a que ésta había quedado inconclusa. ${ }^{8}$ Recibían un salario de 10,000 viejos pesos por trabajar dos semanas cada mes, además de provisiones de alimento por parte de la comunidad. ${ }^{9}$ Impartín la educación básica, siguiendo el modelo guatemalteco; además de enseñar algún oficio y fomentar las actividades colectivas. Asistían a continuar sus estudios en escuelas de Chiapas, ya fuera primaria o secundaria; ahí les enseñaban además diferentes oficios que posteriormente difundían en su comunidad.

La alfabetización a cargo de promotores, mayas y no mayas, era bilingüe y bicultural. El sistema de enseñanza que seguían era el guatemalteco con el fin de preservar en lo posible su identidad nacional y cultural; para el efecto se apoyaron en la enciclopedia temática guatemalteca. ${ }^{10}$ En una temporada, para invitar a los niños a ser partícipes de la educación, organizaron marchas, acompañadas con música, y disfraces. En general, los niños gozaron de mejores posibilidades de educación en los campamentos de las que tenían en Guatemala. ${ }^{11}$

En 1997, al cierre del Comité Cristiano (como lo señalaré después), el Alto Comisionado de las Naciones Unidas para los Refugiados (ACNUR), se hizo cargo de los alumnos que todavía no terminaban su enseñanza, cerró inscripciones y designó a la Hna. Lucía Jiménez como responsable de la conclusión de dicha tarea. A pesar de la clausura de los cursos, algunos planteles continúan abiertos en Santa Rosa, San Lorenzo, La Gloria y Nuevo Huixcán, financiados por ONGs. ${ }^{12}$

La COMAR, que los primeros años del refugio había dado prioridad a la salud y la alimentación, en 1984, trató de tomar en sus manos la responsabilidad de la educación. Para

8 Eduardo Argüello Figueroa, "La población refugiada guatemalteca en Chiapas y su desarrollo educativo, 1997-1998”, Tesis de Licenciatura en Sociología, Universidad Autónoma de Chiapas, SCLC, Facultad de Ciencias Sociales, Campus III, 1998, p. 93.

9 Ricardo Epifanio Pérez Hernández, "Mayas de Guatemala refugiados. Nuestra organización y participación” en Memoria. Presencia de los refugiados guatemaltecos en México, México, COMAR/ACNUR, 1999, p. 65.

10 Isabel María Martínez Portilla, “La diócesis de San Cristóbal...”, op. cit., p. 78.

11 Refugiados guatemaltecos en la diócesis, op. cit., p. 73.

12 Entrevista a Hna. Lucía Jiménez Escalona, op. cit. 
el efecto desarrolló un comité encargado de inscribir y censar a la población infantil, así como de seleccionar a agentes educativos dentro de la misma población. Al iniciar sus actividades, encontró un rezago educativo considerable, ya que el 53\% de la población escolar se encontraba concentrado en primer grado, con edad promedio de 10 años. En los siguientes dos años, se dedicó a organizar la educación de los refugiados reubicados en Campeche y Quintana Roo. Mientras en Chiapas, donde la población tenía el mayor número de niños, el Comité Cristiano continuó a cargo de ésta. En un informe de actividades de la diócesis, publicado entre 1988 y 1989, se dio a conocer que por entonces se llegaron a tener cinco escuelas de educación preprimaria, otras cinco primarias y dos secundarias; que en total atendían 1,260 alumnos.

Para 1989, la COMAR, con el respaldo de la Secretaría de Educación Pública (SEP), se quedó al mando de la enseñanza y estableció en las tres entidades federativas el sistema oficial mexicano de educación. En ese mismo año se expidieron los primeros 160 certificados. A partir de ahí, la COMAR prácticamente se responsabilizó de la instrucción y pago de los promotores de la educación. En este marco, se acordó que el Comité Cristiano continuara su labor, apoyando la instrucción en lo referente a la preservación de la identidad y la cultura guatemalteca. ${ }^{13}$

Con el fin de mantener la comunicación de los avances educativos de los refugiados, entre 1992 y 1996, el Comité publicaba un folleto informativo interno llamado El Ti, en el cual se difundían mensualmente los logros de cada campamento, traídos por los promotores de cada comunidad a la sede del Comité Cristiano donde se imprimía. ${ }^{14}$

La SEP se comprometió a administrar la elaboración de libros de texto gratuito para apoyar en la enseñanza de: geografía, historia, instituciones y cultura de Guatemala. ${ }^{15}$ No obstante, el Comité Cristiano manifestó su anuencia a que la COMAR asumiera plenamente la educación civil de la niñez refugiada, debido a que dicha Comisión pretendía establecer como única opción, el sistema oficial mexicano. Por otro lado, Vladimir Huaroc, funcionario del ACNUR, señaló que a pesar de la presencia de la COMAR en el ámbito educativo, la iglesia continuaba manteniendo en gran medida el control de la educación de los refugiados. ${ }^{16}$ Hacia 1992, la COMAR señaló 13 Idem.

14 Entrevista a Juan Luis Negrete Fernández, op. cit.

15 Esteban Garaiz, “Los derechos humanos y los refugiados guatemaltecos” en Memoria..., op. cit., p. 120. 
que en Chiapas había 95 escuelas localizadas en 86 puntos de refugio, donde se atendían a 5,425 niños. ${ }^{17}$

\section{Proyectos de desarrollo económico}

El Comité Cristiano impulsó programas de actividades económicas para los refugiados, cuyo objetivo era lograr el desarrollo de la autosuficiencia, preservando su identidad étnica y capacitándolos para el regreso a su patria, cuando las condiciones lo permitiesen. ${ }^{18}$ Con base en el trabajo comunitario, las primeras actividades se comenzaron a poner en práctica en 1982; pero a mediados de este año fueron brevemente interrumpidas por la llegada masiva de guatemaltecos que necesitaban urgentemente asistencia material y psicológica; en 1983 se reanudaron.

El Comité Cristiano promovió y apoyó unos 562 proyectos, entre los que había: cooperativas de consumo, cajas de ahorro y área asistencial; además, se impartían talleres en los cuales se producían artesanías y otros bienes; también se fomentaba el cultivo de hortalizas en común y otros productos agrícolas, así como la crianza animales, entre otros. ${ }^{19}$ Según un informe diocesano de 1989, "En estos proyectos trabajaban 740 promotores y participaban 12,125 socios". ${ }^{20}$

Las cooperativas de consumo eran tiendas de abarrote básico manejadas por los refugiados, para comenzar el Comité Cristiano les otorgaba préstamos que después le pagaban. En Margaritas, Paso Hondo y Tziscao fueron las zonas donde se empezaron a promover. El transporte de mercancía era proveído por el Comité, que prestaba sus camionetas para el traslado de los artículos que eran abastecidos en Comitán. Según la Hna. Lucía Jiménez, dichas cooperativas les fueron funcionales, debido a la adecuada organización que tenían.

Entre tanto, en las cajas comunitarias se reunían fondos para las necesidades de los grupos, principalmente se ayudaba a quienes no podían trabajar como: viudas, ancianos y enfermos. En el área asistencial, en 1989, se operaban dos asilos para ancianos y tres orfanatorios, con un

17 Graciela Freyermuth Enciso y Rosalva Aída Hernández Castillo (Comps.), Una década de refugio en México. Los refugiados guatemaltecos y los derechos humanos, México, CIESAS/Casa Chata, 1992, p. 242.

18 "Programas de actividades económicas del Comité Cristiano de Solidaridad” en Boletín, núm.13, México, D.F., Coordinadora de Ayuda a Refugiados Guatemaltecos. Secretaría General, abril de 1986, pp. 10-11.

19 Refugiados guatemaltecos en la diócesis, op. cit., p. 81.

20 Idem. 
total de 386 beneficiados; también había comedores para niños de los cuales no se tienen datos exactos. $^{21}$

En cuanto a los talleres, es de señalar que el primero que se organizó fue de producción de artesanía textil que cumplió varios objetivos: sirvió como terapia psicológica para las mujeres, víctimas de la violencia en Guatemala, y permitió la obtención de ingresos que les permitían hacer frente a su difícil situación económica. El Comité Cristiano, en coordinación con la Secretaría General de la Coordinadora de Ayuda a Refugiados Guatemaltecos, promovían la venta de estos productos, generalmente entre personas e instituciones solidarias en México y en otros países. En 1983, comenzaron estos talleres con mayor formalidad, introduciendo nuevas técnicas de producción. Pero, al año siguiente, se cancelaron al darse el ataque al campamento El Chupadero, el 30 de abril, que obligó a la reorganización de campamentos e incluso a la reubicación de los mismos.

También hubo talleres de costura en los que en un principio se adaptaba la ropa usada que recibían de la población mexicana y que enseguida comenzó a producir directamente ropa para los refugiados. ${ }^{22}$ Asimismo, se constituyó un taller de calzado que producía zapatos y huaraches, tanto para consumo de los refugiados como para venta general, pero éste no dio resultado pues, debido la humedad del clima, les resultaba más práctico el uso de sandalias de plástico. ${ }^{23}$

De igual manera, se formaron talleres de carpintería en los que se producían muebles para uso comunitario en escuelas, comedores infantiles, ermitas, bodegas, dispensarios médicos y cooperativas de consumo. También se instaló un taller de construcción de marimbas, instrumento que contaba con un alto valor cultural y artístico y que tenía demanda en comunidades y ciudades; cuando apenas se consolidaba éste, su actividad se vio interrumpida, al igual que otros, por el ataque al campamento El Chupadero.

En 1983, se iniciaron también de forma más concreta los proyectos agrícolas con el cultivo de hortalizas. En este aspecto, en la zona de Marqués de Comillas se lograron buenos resultados; sin embargo, a causa de la reubicación iniciada en 1984, se interrumpió la labor en esta zona. Allí, adicionalmente, se construyeron granjas de avicultura, porcicultura, apicultura y un taller para hacer pan, cuya producción era para consumo interno; después de la reubicación,

21 Idem.

22 "Programas de actividades económicas del Comité Cristiano de Solidaridad”, op. cit., p. 13.

23 Entrevista a Hna. Lucía Jiménez Escalona, op. cit. 
las instalaciones quedaron para uso de comunidades mexicanas. ${ }^{24} \mathrm{El}$ campamento La Gloria, fue un claro ejemplo de organización colectiva donde se hizo un pozo y una granja que funcionan hasta la actualidad.

En varios casos, las actividades educativas se combinaban con los proyectos de desarrollo económico. De acuerdo con un promotor de la educación cerca de la escuela donde prestaba su servicio tenían una milpa donde los alumnos sembraban, cosechaban y vendían sus productos con el propósito de tener un fondo monetario para alguna ocasión que hiciera falta, por ejemplo, para festejar el día de las madres, sin tener que pedir a los padres de familia su cooperación. Otro ejemplo es el del campamento El Porvenir, que en 1991, abrió una escuela donde, además de actualizar a los promotores en cuestión de salud (nutrición y partos), capacitaba para el funcionamiento de proyectos de desarrollo económico. ${ }^{25}$

\section{Organización para el retorno}

La organización lograda por los refugiados, entre otras cosas a partir de las actividades promovidas por el Comité Cristiano, les permitió hacer escuchar sus necesidades ante las autoridades mexicanas y guatemaltecas. Esto fue un importante punto de partida para estructurar comisiones para el retorno colectivo y organizado; a través de las cuales se logró establecer el diálogo con el gobierno de su país. Las Comisiones Permanentes de Refugiados Guatemaltecos, comenzaron a formarse desde 1986, impulsadas en buena medida por el Comité Cristiano ${ }^{26}$ y quedaron establecidas en 1988. Las Comisiones Permanentes se constituyeron en un organismo representativo integrado por 70 representantes, elegidos por votación directa de los más de 40,000 refugiados instalados en Chiapas, Campeche y Quintana Roo.

A finales de 1987 y principios de 1988, las Comisiones Permanentes se reunieron con representantes del ACNUR y la COMAR y formaron tres comisiones: una para el diálogo nacional, otra para la defensa de la tierra en Guatemala, y otra más para asuntos internacionales. ${ }^{27}$ Luego de un complejo y largo proceso de negociación entre estas instancias y el gobierno guatemalteco, que para el efecto había creado el 26 de septiembre de 1986, la Comisión Especial de Atención

24 “Programas de actividades económicas del Comité Cristiano de Solidaridad”, op. cit., pp. 14-15.

25 Entrevista a Juan Luis Negrete Fernández, op. cit.

26 “Presentación” a Boletín, México, D.F., núm.18, Coordinadora de Ayuda a Refugiados Guatemaltecos.

Secretaría General, septiembre de 1988, p. 3.

27 Idem. 
a Repatriados (CEAR). ${ }^{28}$

En marzo de 1989, a tres meses de iniciado en Chiapas el gobierno de Patrocinio González Garrido, caracterizado por una política altamente represiva, nació el Centro de Derechos Humanos Fray Bartolomé de las Casas, A.C. (CDHFBC) conducido por Samuel Ruiz, al que le correspondió defender ocasionalmente a los refugiados. ${ }^{29}$

En abril de 1990, el Comité Cristiano participó en el foro: “Los refugiados guatemaltecos y los derechos humanos”, ${ }^{30}$ organizado por la Academia Mexicana de Derechos Humanos $(\mathrm{AMDH})$ y el Centro de Investigaciones y Estudios Superiores en Antropología Social del Sureste (CIESAS), realizado en San Cristóbal de las Casas. Además del Comité participaron representantes del ACNUR, la COMAR y las Comisiones Permanentes, entre otros. En el foro se trataron cuatro temas: el derecho al refugio, a la cultura, a la salud, al retorno. Se planteó la necesidad de incorporar a los refugiados al sistema mexicano, debido a que en Guatemala aún persistía la violencia; en el caso del retorno voluntario se insistió en reglamentarlo jurídicamente a nivel internacional. La clausura estuvo a cargo del obispo de San Cristóbal. ${ }^{31}$

Es de anotarse que en medio del proceso de negociaciones, el 15 de agosto del mismo año, se constituyó la agrupación de mujeres guatemaltecas refugiadas llamada Mamá Maquín, en el campamento La Cieneguita, donde participaron 700 mujeres como en las Comisiones Permanentes, representantes de campamentos y asentamientos de las tres entidades federativas mexicanas donde había refugiados. Al acto acudieron más de 300 invitados, entre ellos hubo representantes de: las propias Comisiones Permanentes, el Comité Cristiano, el Hospital General de Comitán, la COMAR, el ACNUR, el UNICEF, ONGs, Acción Permanente Cristiana por la Paz, La Doble Jornada, Radio Educación, etc.; desde Guatemala llegaron varias representaciones, entre ellas: la Coordinadora Nacional de Viudas de Guatemala (CONAVIGUA), y la Acción Cristiana Guatemalteca (ACG); de El Salvador asistió la Congregación de Madres Cristianas por

28 Carmen Rosa de León Escribano, "Repatriación voluntaria. Creación de la CEAR” en Memoria..., op. cit., pp. 155.

29 Cuadriello Arriagada, Mario, “Golpe de timón en la nave de la iglesia. Ascenso, auge y declive del obispo Samuel Ruiz”, México, Tesis de Licenciatura en Relaciones Internacionales en el Centro de Estudios Internacionales de El Colegio de México, México, mayo de 2003, pp. 98-100; Fazio, Carlos, Samuel Ruiz. El caminante, México, Espasa Calpe, 1995, p. 169.

30 "Refugiados" en Resumen informativo, núm. 42, SCLC, Centro de Información y Análisis de Chiapas (CIACH), abril de 1990, p. 33.

31 Los refugiados guatemaltecos y los derechos humanos. Cuaderno para refugiados guatemaltecos, Chiapas, México, Gobierno del Estado de Chiapas, Instituto Chiapaneco de Cultura, 1991, pp. 2-15. 
la Paz; también llegaron observadores de España, Estados Unidos y Alemania. ${ }^{32}$ Mamá Maquín recibió apoyo de: religiosas del Comité Cristiano, ACNUR, Centro Integral de Apoyo a la Mujer (CIAM) y ONGs.

Entre los principales objetivos de Mamá Maquín estaban: exigir la igualdad de derechos, de participación y de decisión entre mujeres y hombres, construir la democracia en las comunidades y en Guatemala, alcanzar un retorno digno y seguro, y lograr el respeto a las culturas indígenas. Intensificó el trabajo en talleres, programas de radio, reuniones de mujeres, etc., con el propósito de dar a conocer la situación de Guatemala, ${ }^{33}$ para 1999, agrupaba a más de 8000 mujeres guatemaltecas.

El 8 de octubre de 1992, fueron firmados los acuerdos para el retorno en los que quedaron establecidas seis condiciones básicas: 1) decidir voluntaria e individualmente el retorno; 2) garantía de regreso a sus tierras; 3) reconocimiento del derecho a organizarse y asociarse libremente; 4) respeto a la integridad personal y comunitaria; 5) regresar acompañados por ONGs nacionales e internacionales y representantes del ACNUR; y 6) garantía de libre movilización nacional e internacional de los que regresaran. ${ }^{34}$ Después de la firma de los acuerdos de 1992, las Comisiones Permanentes comenzaron a planear cómo se haría el retorno, y a qué puntos regresarían.

Cabe destacar que, con el fin de mantener informados de los avances obtenidos por cada delegación de las Comisiones Permanentes, el Comité Cristiano financió y difundió una publicación periódica llamada Nuevo Día, que circuló aproximadamente cinco años, casi los mismos que abarcó el proceso de retorno.

Finalmente, en enero de 1993, se dio el primer retorno organizado de guatemaltecos a su patria. Después se dieron los siguientes: el de las cooperativas del Ixcán, en diciembre de 1993; el de Chaculá, en enero de 1994; y el de Fray Bartolomé en noviembre de ese mismo año; a ellas siguieron los de Momonlac, Ixtahuacán Chiquito, San Antonio Tzejá y el de Chancolín, en 1995; y otras más para cerrar el ciclo, en $1998 .{ }^{35}$ Cada regreso implicó la exigencia, ante el gobierno guatemalteco de créditos y de recuperación de las tierras de los que regresaban. Desde el primer

32 Fernando Solís Cámara, “Presentación” en Memoria..., op. cit., pp. 11-15.

33 Carlos Véjar Ramos, “La participación tripartita en el proceso de retorno colectivo” en Memoria..., op. cit., p. 210.

34 Hermitaneo Monzón, “Camino a los acuerdos. Del 8 de octubre de 1992” en Memoria..., op. cit., pp. 166-169.

35 Mercedes Oliveira, et. al., Nuestra experiencia ante los retos del futuro...”, op. cit., pp. 38-39. 
retorno, Mamá Maquín tuvo una integrante en cada camión, formó una red de información sobre la ruta, las paradas, los trámites, etc. que se debían hacer para el regreso a Guatemala.

En cada caso, Mamá Maquín jugó un papel importante. Con la organización para el último retorno se cerró el ciclo de la relación entre el Comité Cristiano y los guatemaltecos, abierto desde la llegada de los primeros refugiados. El Comité se clausuró un año antes, en 1997, pero los que decidieron quedarse en México, estuvieron apoyados por las siguientes organizaciones: Educación para la Paz (EDUPAZ), con sede en Comitán, dirigido por maristas; y Cáritas México con una sede en el seminario de San Cristóbal de las Casas. Hoy en día continúa la labor de estas dos organizaciones y su atención se ha extendido a la población chiapaneca.

Como se puede apreciar, frente a la ambigüedad del gobierno mexicano hacia los refugiados guatemaltecos, la diócesis de San Cristóbal de las Casas desarrolló un importante papel en esa región a través de su Comité Cristiano, en el que se destacó la labor de los promotores, quienes fueron preparados para atender a sus compatriotas en cuestiones de salud y educación. Asimismo, el Comité promovió programas de desarrollo económico encaminados hacia la autosuficiencia, también estuvo al pendiente en cuanto a la organización para el retorno apoyando a las Comisiones Permanentes de Refugiados Guatemaltecos y al movimiento de mujeres refugiadas Mamá Maquín. Otra labor de importancia del Comité Cristiano fue la difusión, a través de boletines informativos sobre el acontecer general de los campamentos, la educación y el retorno; entre los boletines se destacaron: El Caminante, El Ti y Nuevo Día, los cuales contribuyeron a que se difundiera a nivel nacional e internacional la cuestión del refugio guatemalteco en México. Tales publicaciones contenían denuncias, la mayoría de las veces expresadas en cartas colectivas, generaron a la diócesis y a sus miembros amenazas y secuestros por parte de los gobiernos de ambos países.

Esimportantedestacarlaparticipación dedospublicaciones quetambién daban seguimiento al caso: Resumen informativo, que editaba el Centro de Información y Análisis de Chiapas (CIACH); y el Boletín, publicado por la Coordinadora de Ayuda a Refugiados Guatemaltecos en el Distrito Federal. Esta difusión obligó a los gobiernos mexicano y guatemalteco a tomar partido en el asunto, representados por la COMAR y la CEAR, respectivamente. 